\title{
Why socially assistive robots?
}

\section{Juan Pedro Bandera Rubio}

6th World Convention on Robots, Autonomous Vehicles and Deep

Learning

September 10th, Singapore 


\section{Index}

TecnologíaElectrónica

- A new generation of robots

- SAR

- Are SAR really useful?

- Designing SAR from the very beginning

- Use case: the CLARC robot

- Conclusions

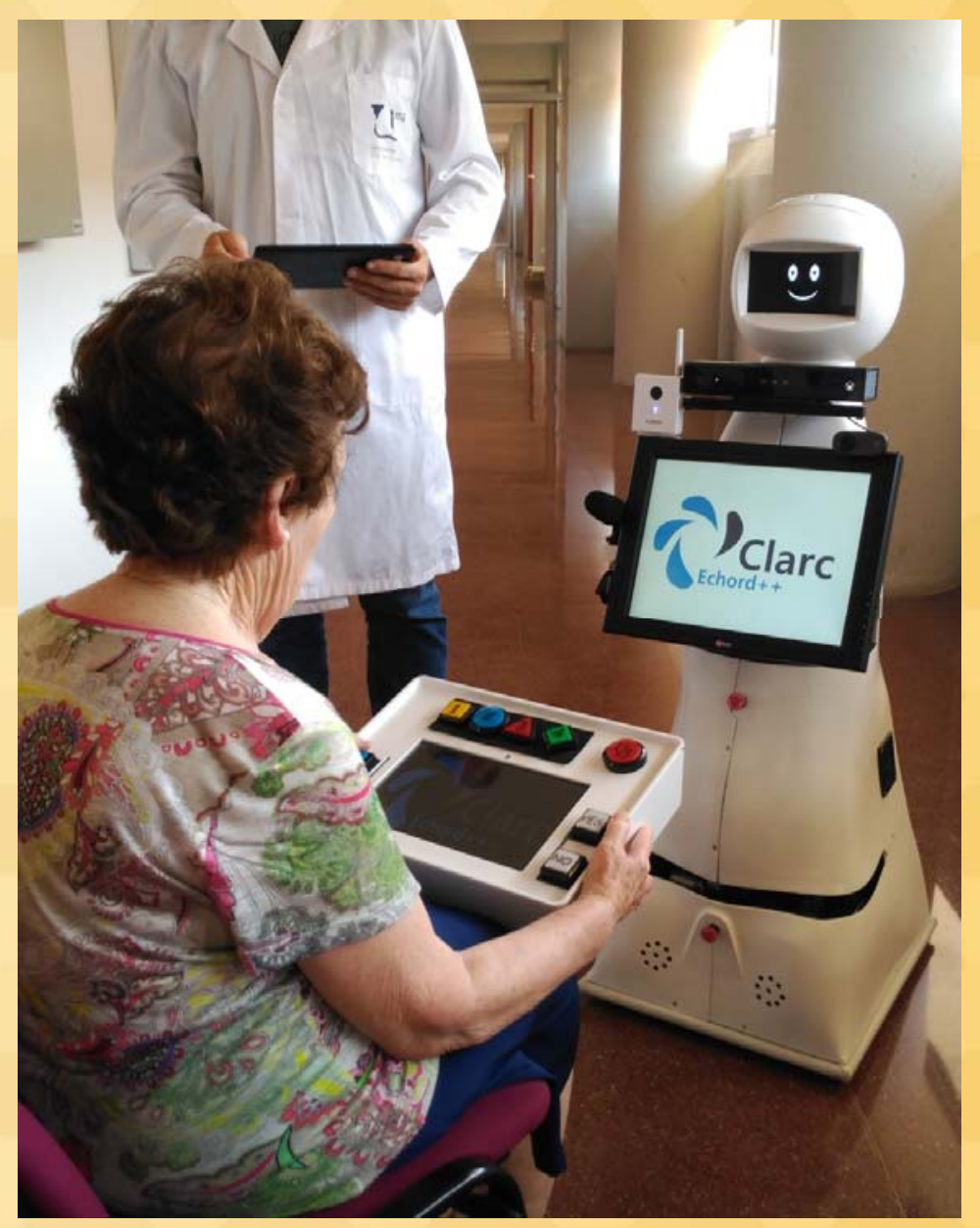




\section{Index}

AVISPA

TTE竞

TecnologíaElectrónica

- A new generation of robots

- SAR

- Are SAR really useful?

- Designing SAR from the very beginning

- Use case: the CLARC robot

- Conclusions

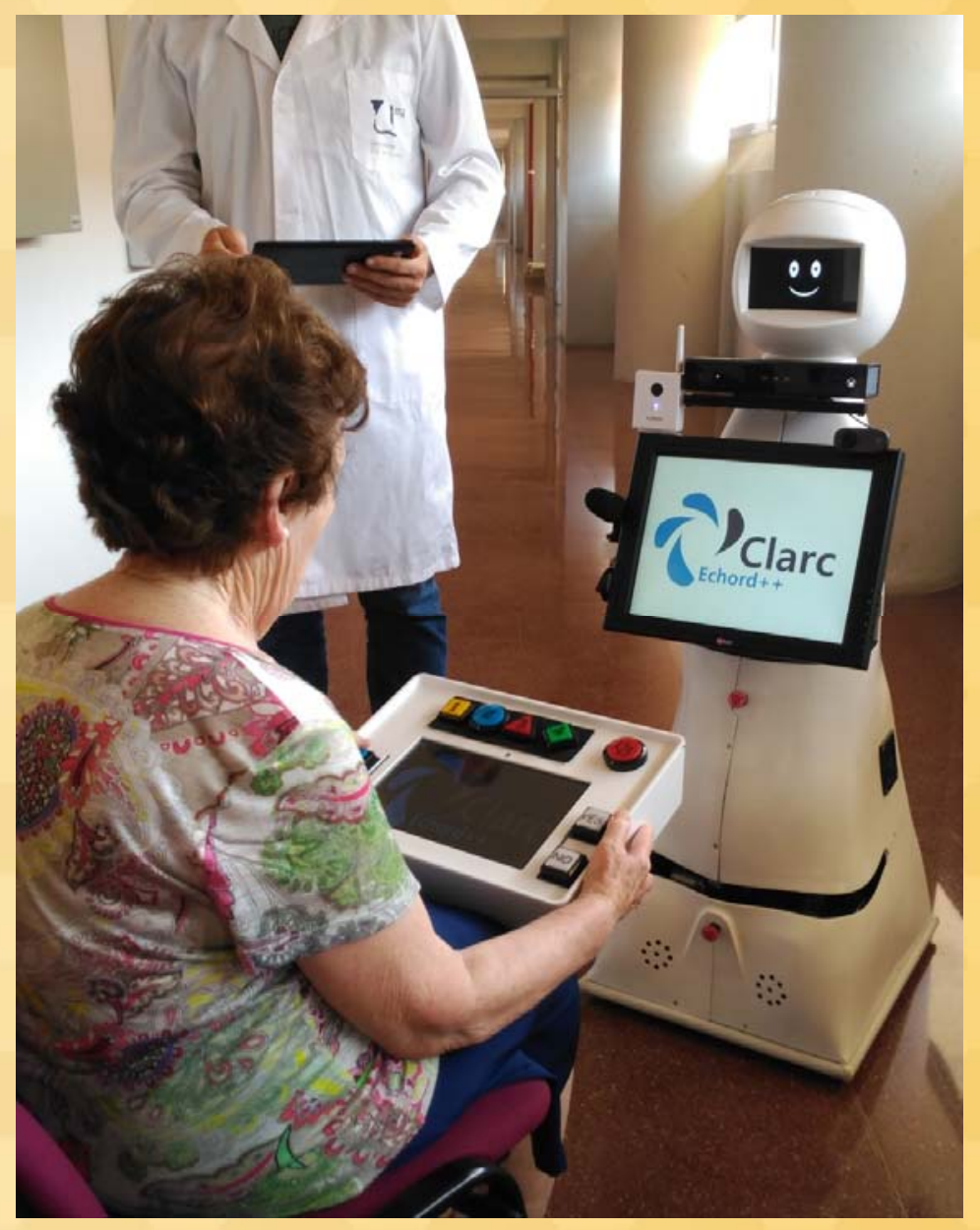




\section{Current robot applications}

- Traditional classification:

- Industrial robots

- Service robots

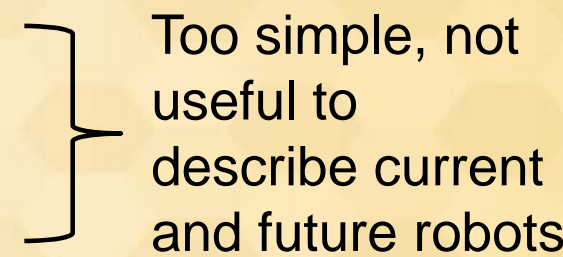

- New industrial robots:

- Co-operative, interactive

- Mobile, autonomous

- Flexible, adaptable (learn)

- They are used for manufacturing...

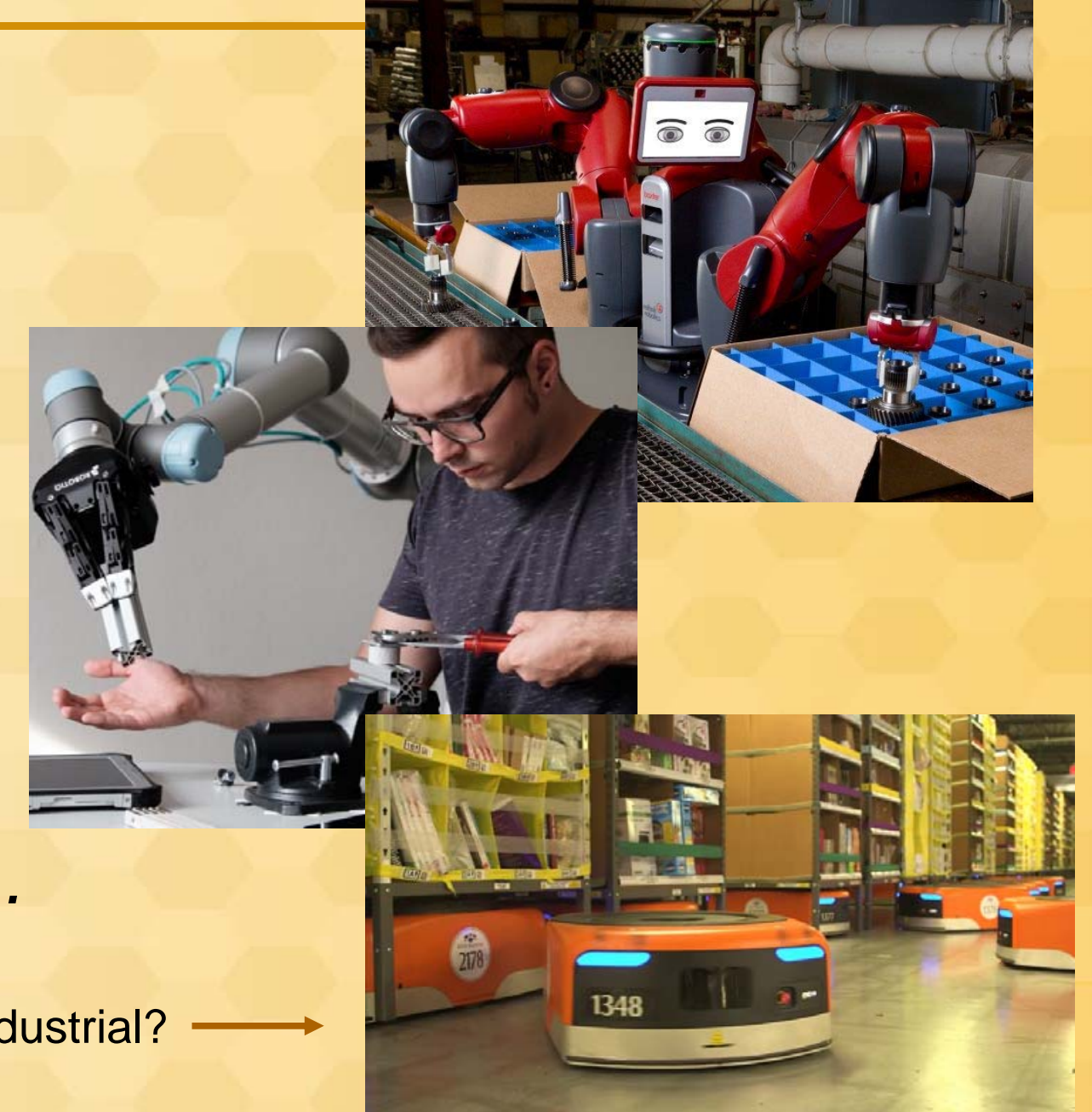




\section{New robots for new applications}

TecnologíaElectrónica
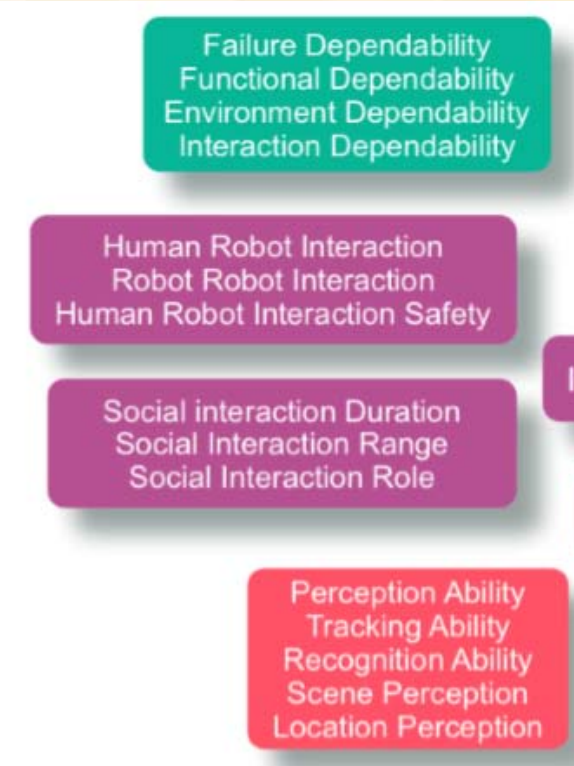

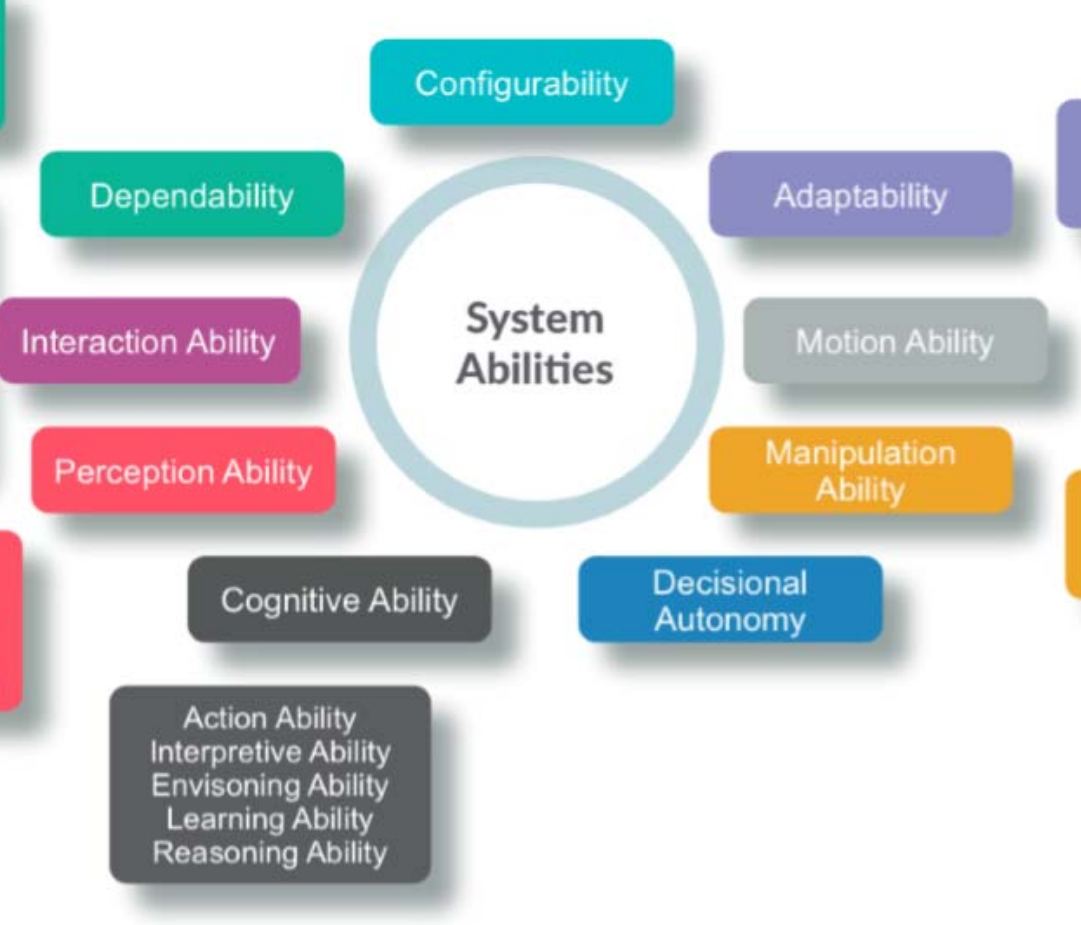

Parameter Adaptability

Component Adaptability Task Adaptability

Constrained Motion

Unconstrained Motion

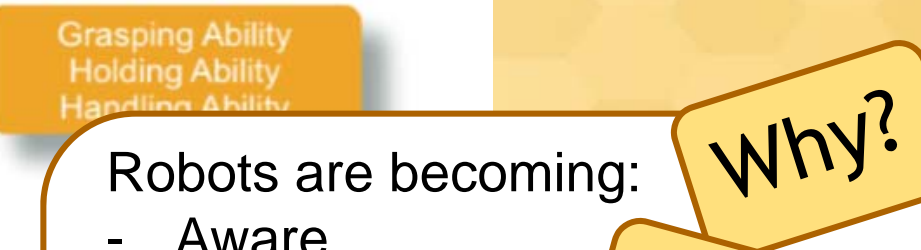

- Aware

- Cooperative

- Autonomous

- Cognitive

- Adaptive

- Proactive
Taken from:

SPARC: The partnership for robotics in Europe. Robotics 2020 multi-annual roadmap for robotics in Europe.

The EU framework programme for research and innovation. Report. 2015

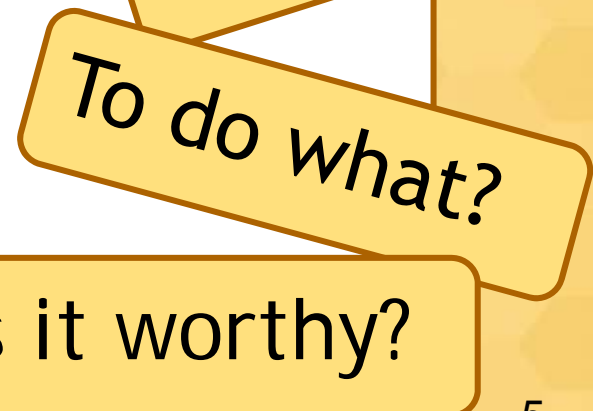




\section{Roadmap for robot applications}

TecnologíaElectrónica

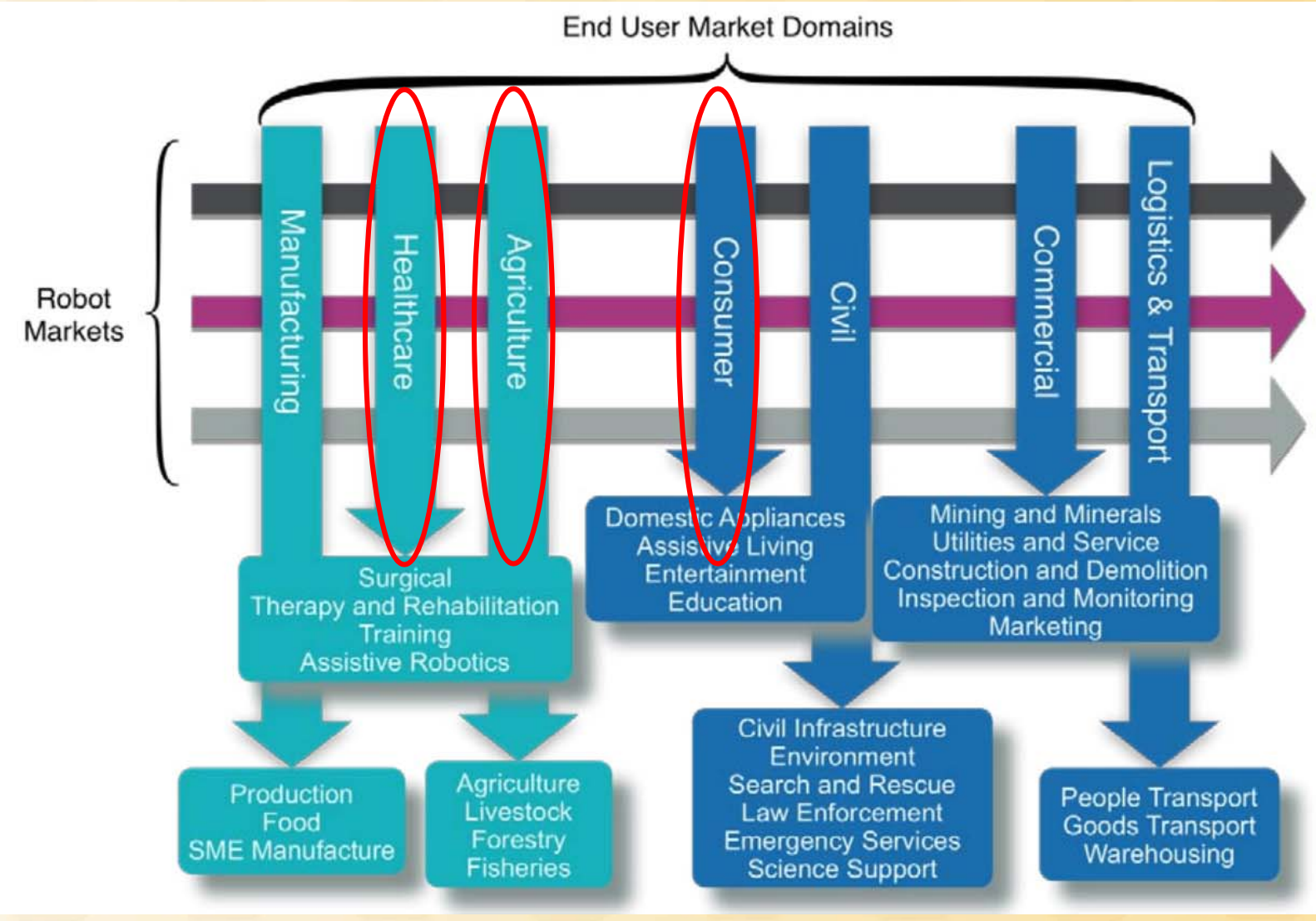

Technologies

Taken from:

SPARC: The partnership for robotics in Europe. Robotics 2020 multi-annual roadmap for robotics in Europe.

The EU framework programme for research and innovation. Report. 2015

Robotics as a tool to face demographic

Why socially assistive robots? Juan Pedro Bandera Rubio changes 


\section{The silver society}

TecnologíaElectrónica

\begin{tabular}{|c|c|c|}
\hline & 2015 & 2030 \\
\hline Andalucia & $19.6 \%$ & $25.8 \%$ \\
\hline Spain & $18.2 \%$ & $24.9 \%$ \\
\hline Europe & $18.3 \%$ & $24 \%$ \\
\hline Japan & $25.9 \%$ & $30,4 \%$ \\
\hline
\end{tabular}

$\%$ population $>64$ years

- 2035: There will be more people aging 64 or more, than people in working ages

- Less caregivers, more medical and social expenses 


\section{The silver society}

\section{Requires measures}

- Active ageing

- Help seniors maintain their functional ability

- Healthy living, healthy ageing

- Include medical equipment at home

Let the elderly people live as autonomous as possible, as long as possible

- Improve hospitals, nursery houses and day care centers

- with new tools to ease the daily work of healthcare professionals

New technologies, including robotics 


\section{Medical robots}

- Clinical robots:

- Diagnostic or surgical assistance. Teleoperation

- Minimally-invasive surgery

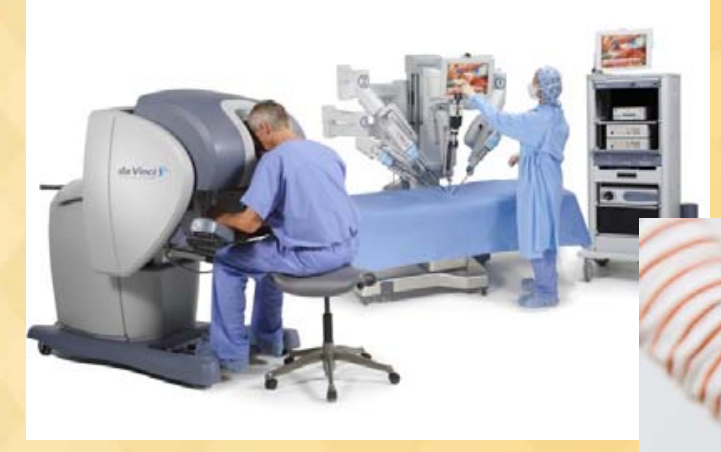

- Rehabilitation robots:

- Prosthesis, exoskeletons, motion help and monitoring systems...

- Specialist supporting assistant robots:

- Help in daily care routines, such as standing up, sitting down, going to the toilet, etc.

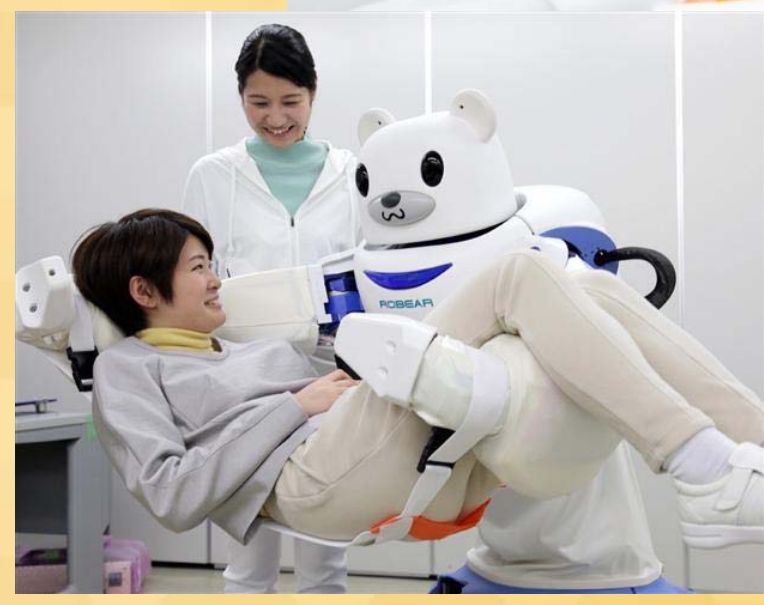




\section{Assistive Robots}

Assistive robot: one that helps a human user

- Autonomous wheelchairs, robotic manipulators, companion robots...

- Key difference respect to medical robots: working scenarios

- Daily life environments, not necessarily medical facilities

- Constant supervision not required

- Non invasive assistance

- Devices more interactive and more versatile

- Consumer devices

- Conceived as a part of a smart environment, in which they cooperate with other technologies, such as other assistive robots or smart houses 


\section{Socially Assistive Robots}

We define socially assistive robots (SAR) as the intersection of assistive robots $(A R)$ and socially interactive robots (SIR). SAR shares with assistive robotics the goal to provide assistance to human users, but it specifies that the assistance is through social interaction. (Feil-seifer y Mataric, 2005)
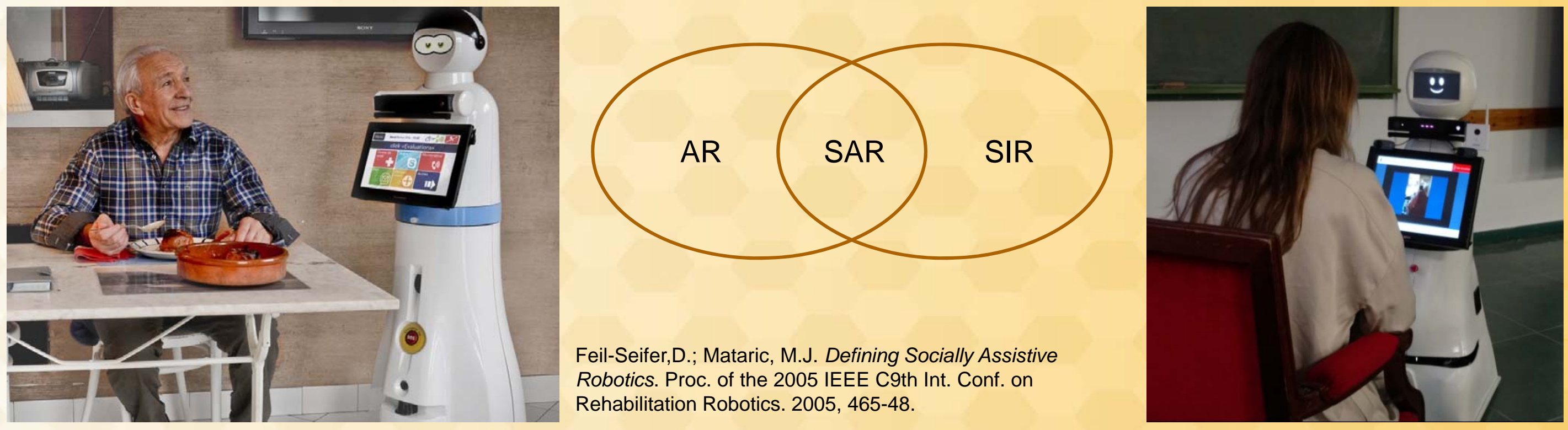

Feil-Seifer,D.; Mataric, M.J. Defining Socially Assistive Robotics. Proc. of the 2005 IEEE C9th Int. Conf. on Rehabilitation Robotics. 2005, 465-48. 


\section{Index}

TecnologíaElectrónica

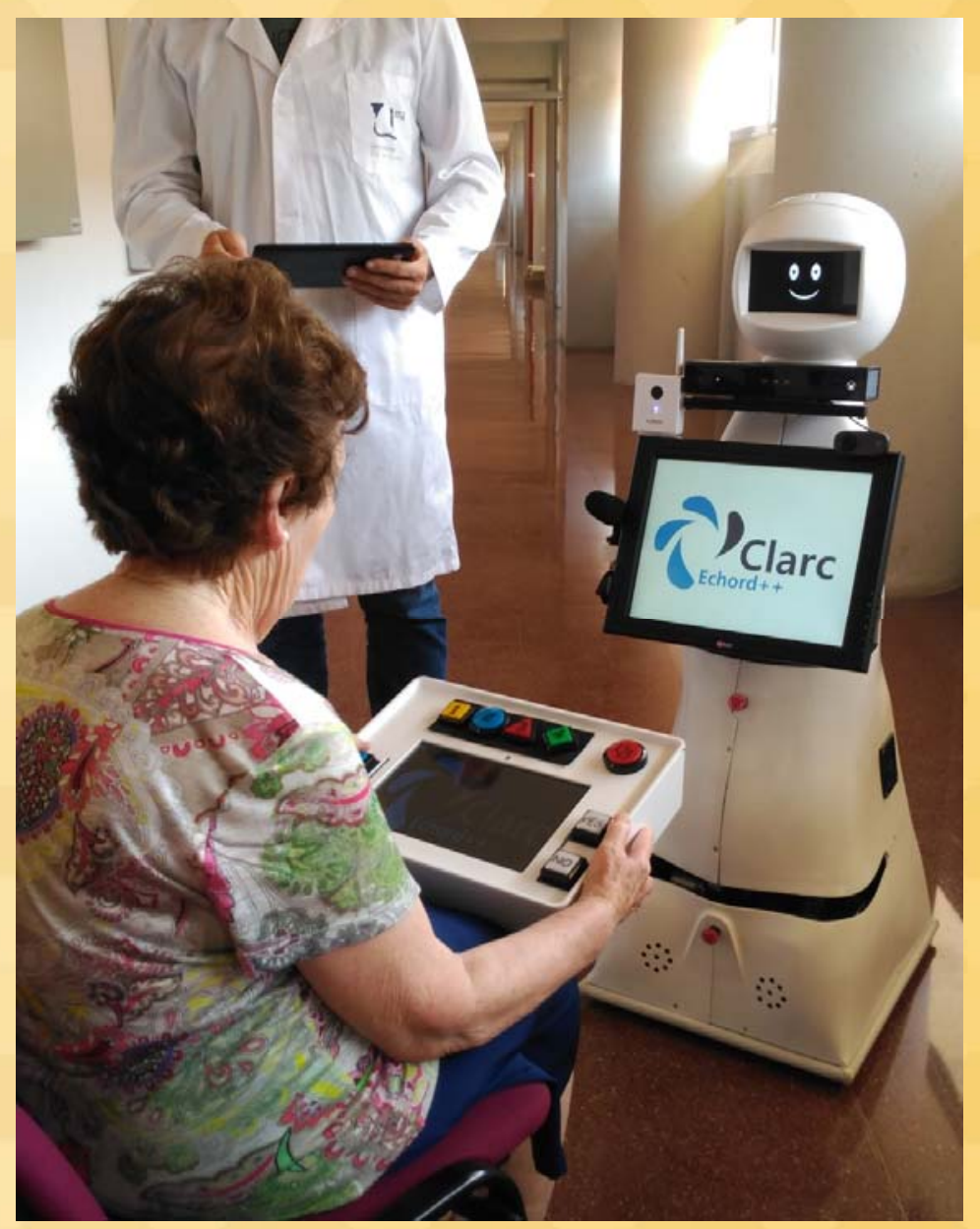




\section{Socially assistive robots (SAR)}

TecnologíaElectrónica

\section{Goal:}

- Assist people Motivation to use them:

- Versatile

- Proactive

- Social interaction is a key part of many therapies

- Embodiment eases acceptability

- No physical contact required Improving tech.

- Attitude

- Usability

- Confidence 


\section{Socially assistive robots (SAR)}

AVISPA

杼E咅

\section{Shared characteristics:}

- Physical

- Safe and social navigation

- Multimodal interaction: robust and accesible

- Expressions: to let the user know about the robot's state (no emotional interaction)

- Passive and active monitorization
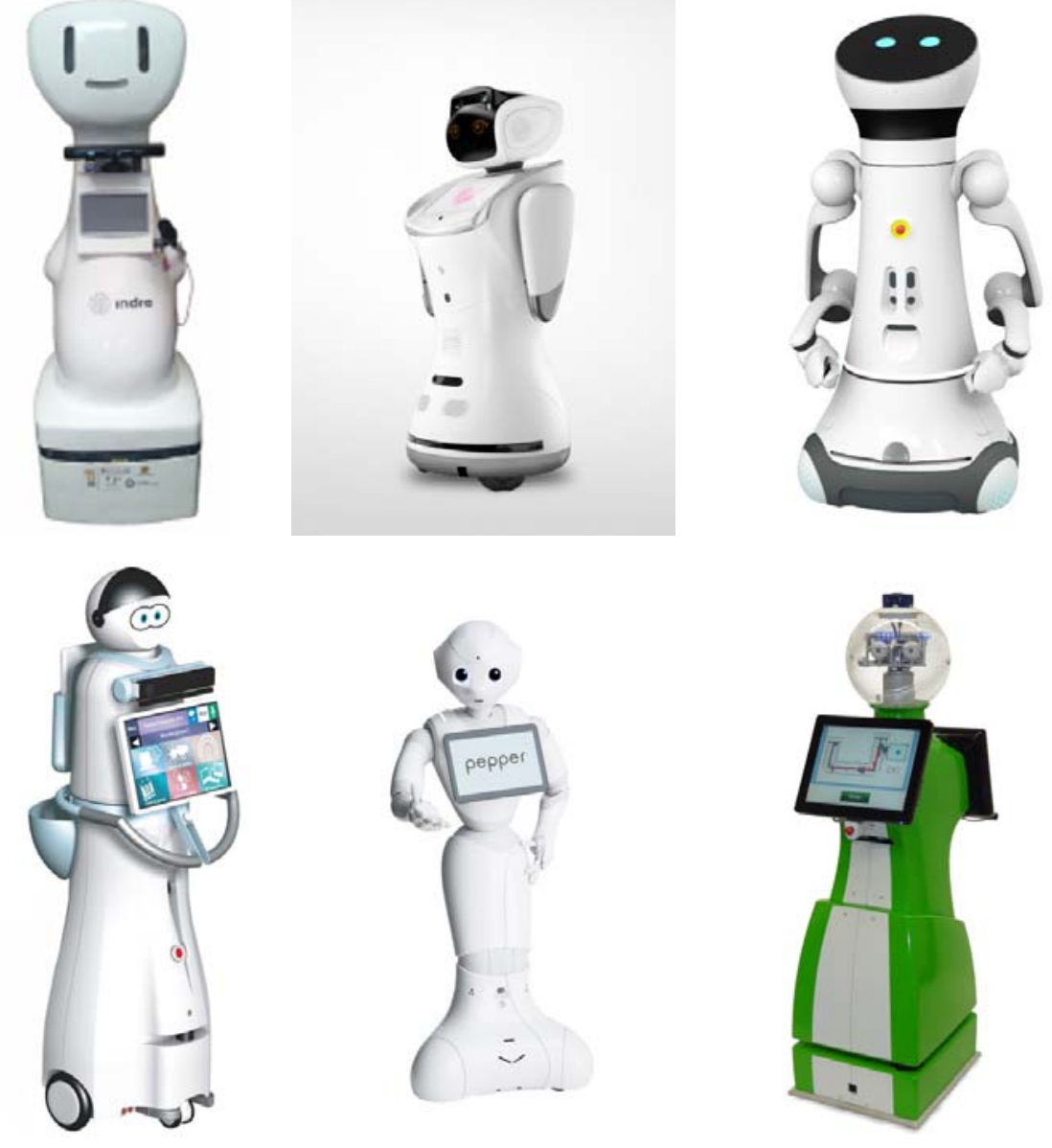


\section{SARs today: A review}

\section{Relevance:}

- Important inversion

- Many projects: Accompany, HOBBIT, SILVER, SERROGA, Florence, Mobiserv, Robot-ERA, ROREAS, SIRMAVED, CLARC, Assesstronic, ...

- Great potential for applications

- Relevant business opportunity

\section{Results:}

- Useful: They can motivate, monitorize, inform...

- efficiently, and in a non-invasive way

- User's overall opinion: Good qualitative results

- Helps maintaining an independent living

- Stress reduction

- Improves mood

Short

-term

- Decreases loneliness

15 


\section{Index}

TecnologíaElectrónica

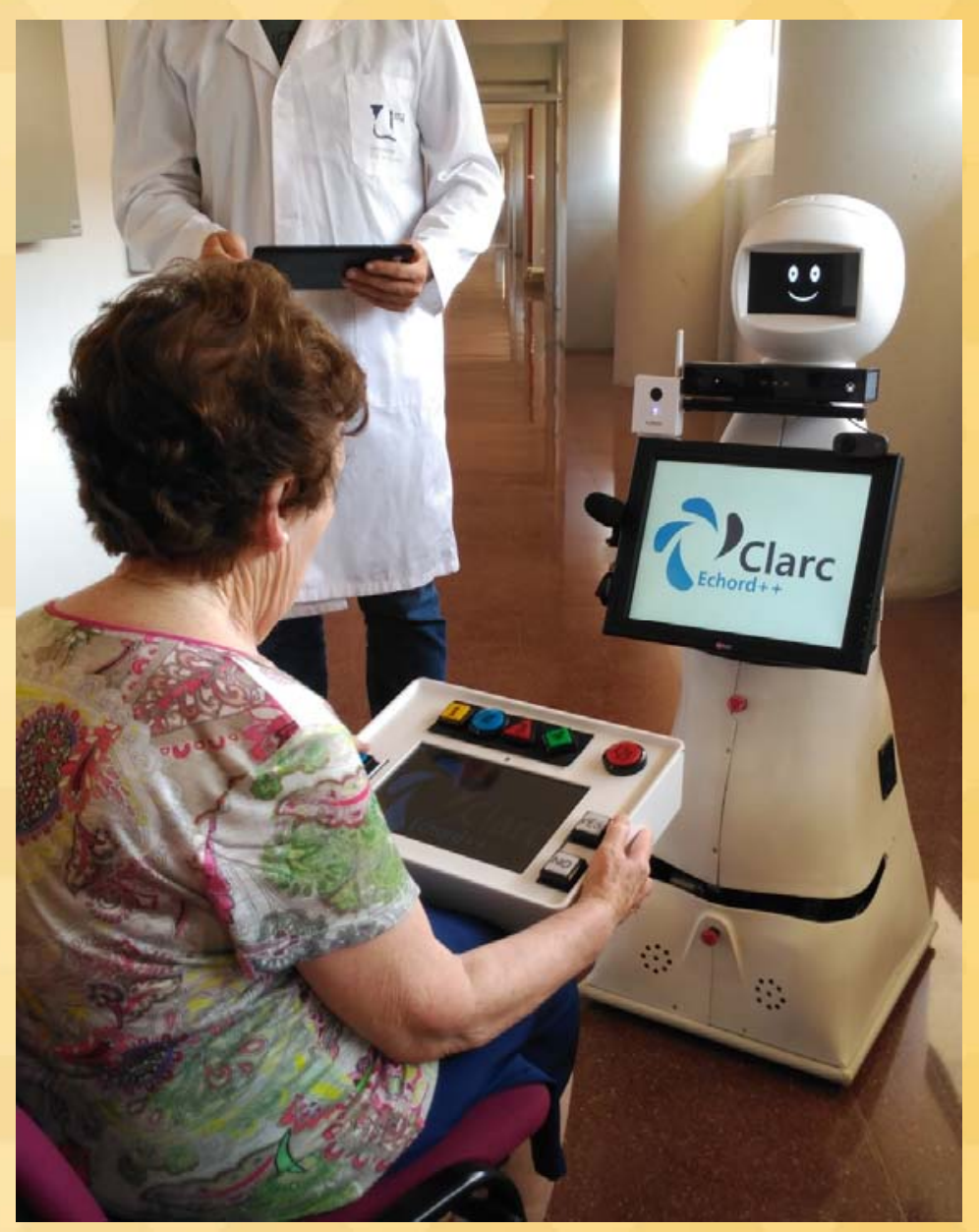




\section{Two real examples of SAR}

TecnologíaElectrónica

- Project \#1

- Funding (total): 4,8M EUR

- SAR purpose:

- Entertainment

- Cognitive and social assistance

- Physical support for retrieving or transporting items

- SAR employed: Expensive

- Working scenario: Homes
- Relies on increasing acceptability via affection and emotions.

- Experiments: Two elderly people used the robot at home for three weeks.

- Results:

- Technically correct

- Potentially interesting tool

- Show that after novelty effect the seniors didn't adhere very much to the robot 


\section{Two real examples of SAR}

TecnologíaElectrónica

- Project \#2

- Funding (total): 4,1M EUR

- Started as an effort to identify new technologies able to assist people.

- SAR purpose: Ease independent living

- SAR employed: Robotic walker.
- Working scenario: Home, day care centers, hospitals, ...

- User-centered designed.

- Designed to be useful and accepted via accesibility, usability and confidence

- Results:

- The robot is currently on the market 


\section{What are SAR doing well?}

TecnologíaElectrónica

- Work autonomously

- Interact with Smart Environments

- Positive initial response

- Adapt to different roles, tasks and scenarios

- Not better than a computer game for cognitive training...

- Not much better than a pet or toy to reduce stress in one-to-one interactions...

- Not better than other devices to monitor people...

- But one SAR can do all these tasks and more
- They are very good social facilitators

- Best when they are included in a group of people

- Best scenarios: Retirement houses, daily care centers, hospitals, ...

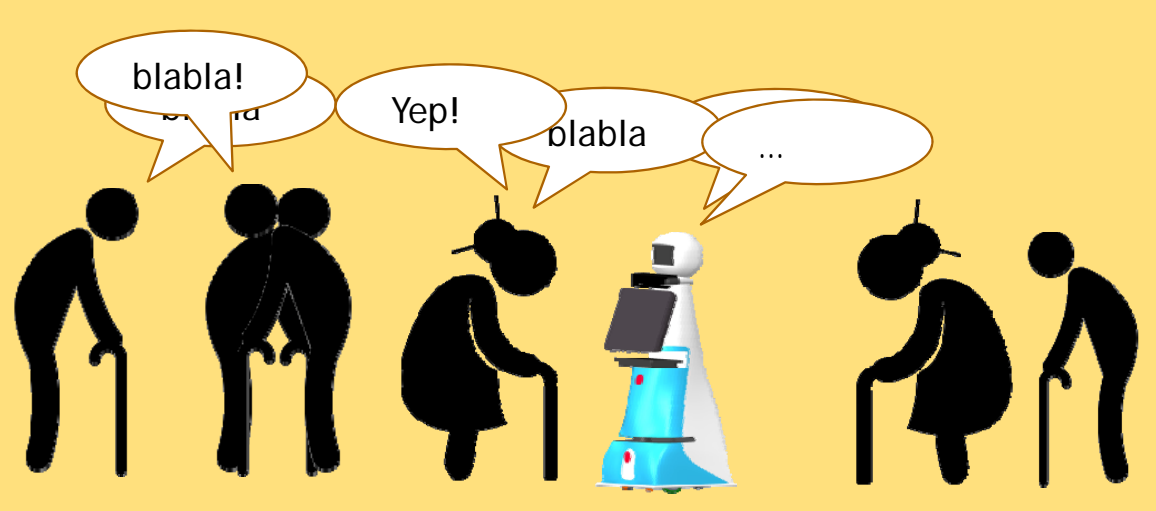




\section{What's wrong?}

TecnologíaElectrónica

- Limited experimentation. Common issues (Broekens et al. 2009, Mataric 2017):

- Control group

- Reproducible experiments

- Long term experiments (months, or years!)

- Studies should cover a significant sample of the target population

- Broekens, J.; Heerink, M.; Rosendal, H. Assistive social robots in elderly care: a review. Gerontechnology. 2009; 8(2):94-103.

- Mataric, M.J. Socially assistive robotics: Human augmentation versus automation. Science Robotics. 2017; 2. 


\section{What's wrong?}

TecnologíaElectrónica

More commonly detected issues:

- Uncanny valley:

- False expectations

- Boredom:

- No real effects in daily routines,

- Feeling that the robot is not of real use

- Not useful for healthcare professionals:

- If it doesn't Save time, if it doesn't provide new really useful features...

- WHY USING THEM?

- Many current SAR are not correctly designed, are not adequately tested in real scenarios, are not really useful, and/or are not being used in adequate applications.

- They cannot be a 'promising' device forever. It's time to begin creating really useful SAR.

- How can we do so? 


\section{Index}

TecnologíaElectrónica

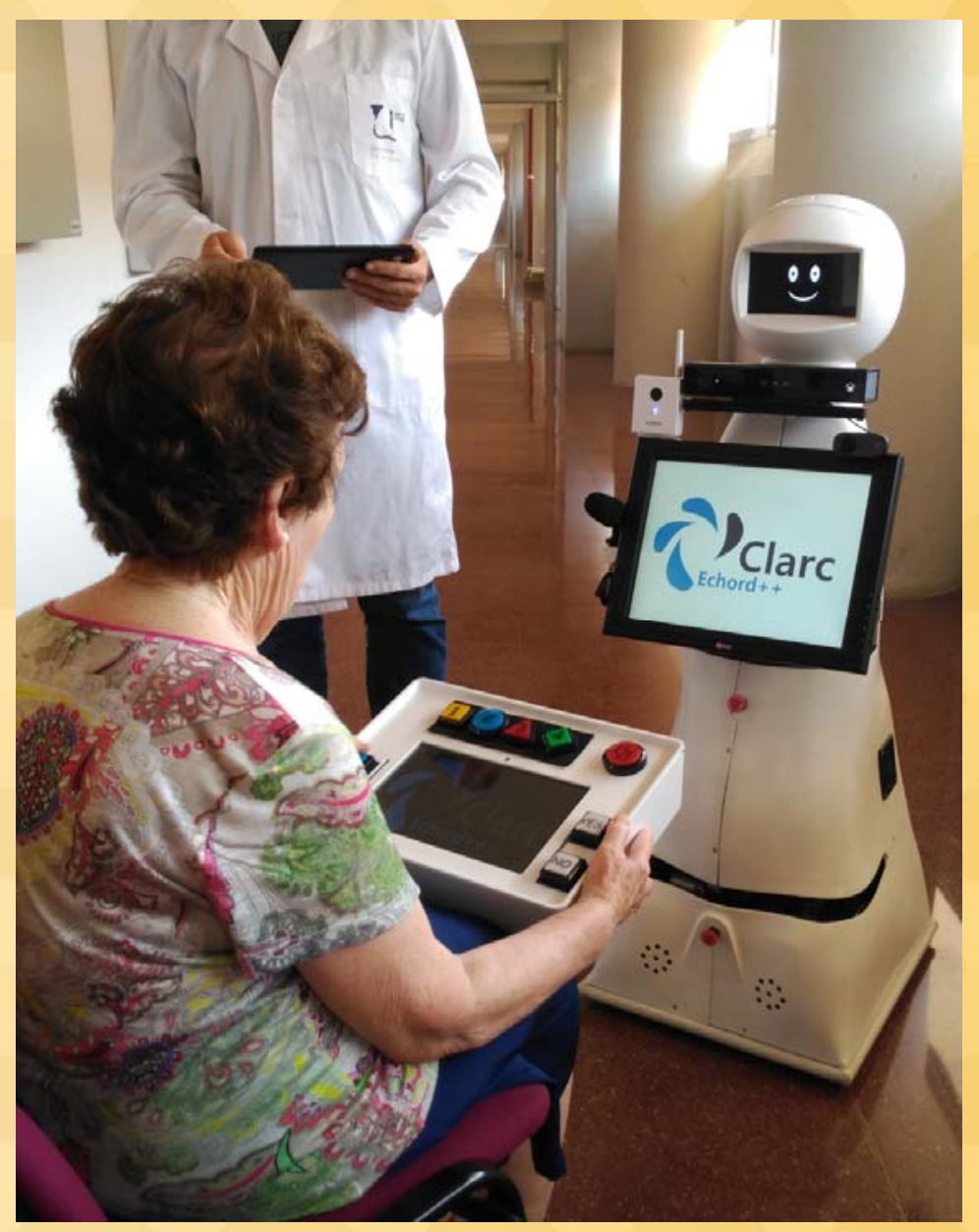




\section{User centered design}

TecnologíaElectrónica

- Count with the users in all stages of the design process

- Which users / stakeholders? ALL.

- For instance, for a SAR working in a retirement house:

\begin{tabular}{|l|l|}
\hline Primary & Elderly people, caregivers \\
\hline Secondary & Family, friends, visitors, external healthcare professionals \\
\hline Tertiary & Rest of workers, management staff, external hw \& sw providers \\
\hline
\end{tabular}

- Matches with prototyping models

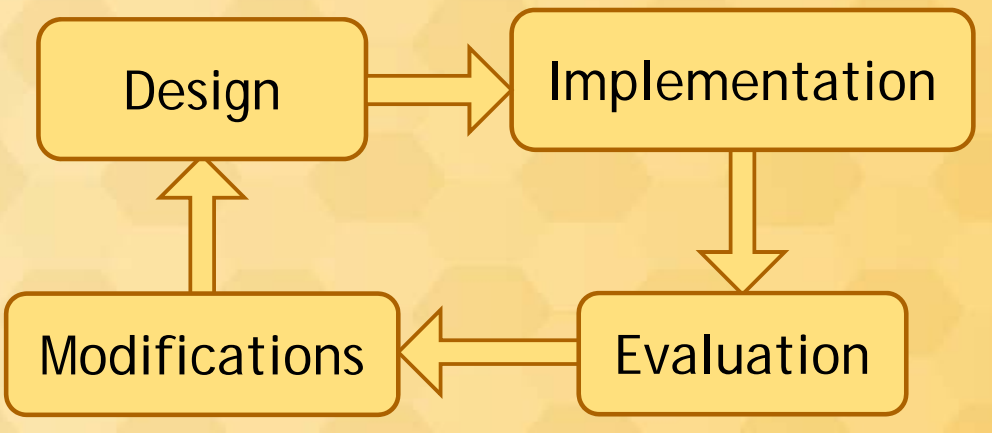




\section{Listening to the elderly people}

TecnologíaElectrónica

Elderly people is a wide target! In general:

- Cultural issues

- They are positive towards the

- Use of technology

- Expectatives

- Cognitive issues
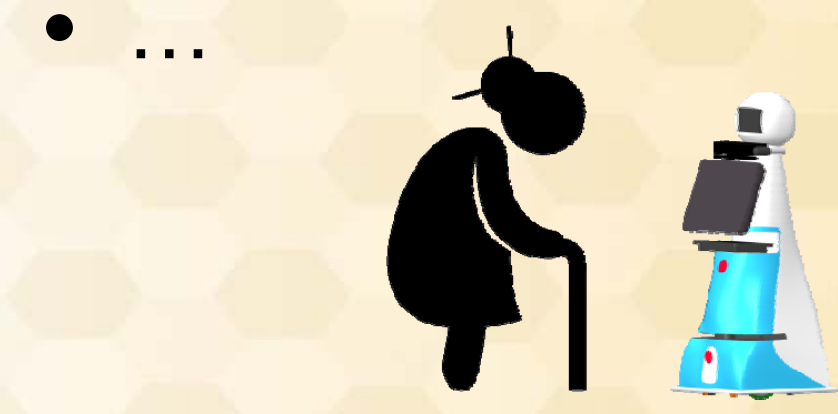
robot

- False expectations are very harmful for adherence

- Functionality is the key component for long-term acceptance

- Attitude, Usability, Confidence 


\section{Listening to the caregivers}

TecnologíaElectrónica

Also a wide target

- Cultural issues

- Use of technology

- Expectatives

- Concerns

- ...

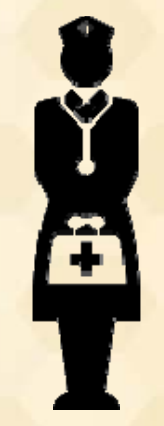

In general:

- Worried about robots that seem to be designed to replace or monitor them

- Happy if the robot makes their job easier

- Saving time, easing tasks...

- Utility is the key component for long-term acceptance 


\section{Listening to the manager}

TecnologíaElectrónica

\section{Why using a SAR?}

- Catch attention of potential clients

- Monitor people

- Careful!

- Provide new functionalities

- Affordable cost

- Careful!

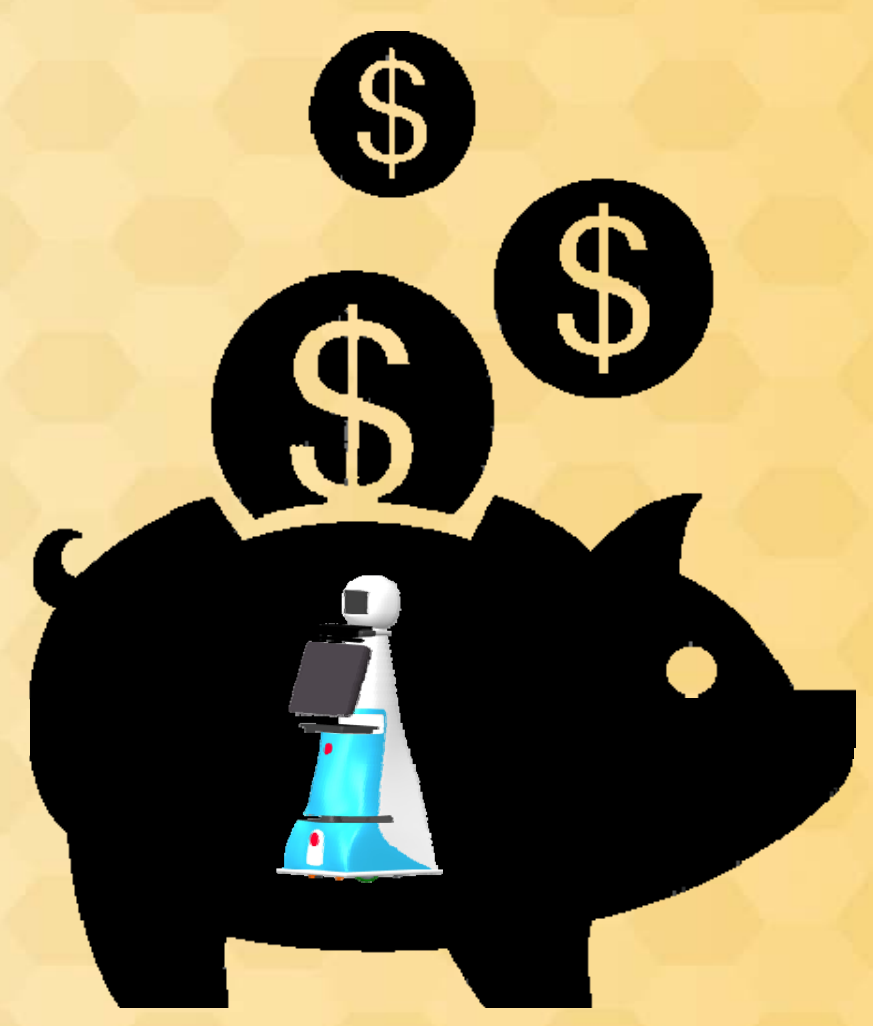




\section{Listening to the engineers}

TecnologíaElectrónica

\section{What's "easy" for a SAR?}

- Connect to smart environments

- Carry tactile interfaces

- Speak

- Monitorize

- Detect people

- Avoid collisions when navigating and trace reasonable routes

- Autonomous navigation can be considered a solved problem

\section{What's complex?}

- Recognize emotions

- Free talking

- Listen

- Social navigation

- Manipulate in unconstrained scenarios

- Imagine, prevent,

$\bullet \quad \ldots$ 


\section{Listening to the engineers}

Main requirement: Make a set of specifications for the SAR

- Adaptability VS (Cost \& Robustness)

- The more you ask, the more it costs

- So... what do you really need the SAR to do? Do you really need < arms / complex expressions / hand gesture recognition >?

\section{Safety concerns}

- ISO 13482:2014 standard for service robots: Not enough... not widely used

- Current common trick: Compliance of all components makes the total machine compliant

- Careful! 


\section{Ethics}

TecnologíaElectrónica

\section{SAR Goal:}

- Assist people: Two different meanings are posible

- Become an autonomous assistant that can replace a human caregiver

- Become a tool for caregivers to help assist people more efficiently 


\section{Ethics}

TecnologíaElectrónica

\section{SAR Features:}

- A SAR should not look like human

- The more anthropomorphic a SAR is, the more expectations it produces.

False expectations have to be avoided at all cost!!!

- Ethical risk of identification and confusion is greater in the target population (elders with possible cognitive impairments)

- Same applies to emotional stuff: The less emotional the SAR is, the better. 


\section{Ethics}

TecnologíaElectrónica

\section{SAR behaviours and legal issues}

- A SAR is a machine. It is not a person, not even from a strictly legal point of view.

- Who is responsible?

- Designer or maker

- Open source: Hw or Sw maker

- Supervised learning: The user who taught

- Autonomous learning

The 'electronic person' concept is not a legal not an ethical option 


\section{Index}

TecnologíaElectrónica

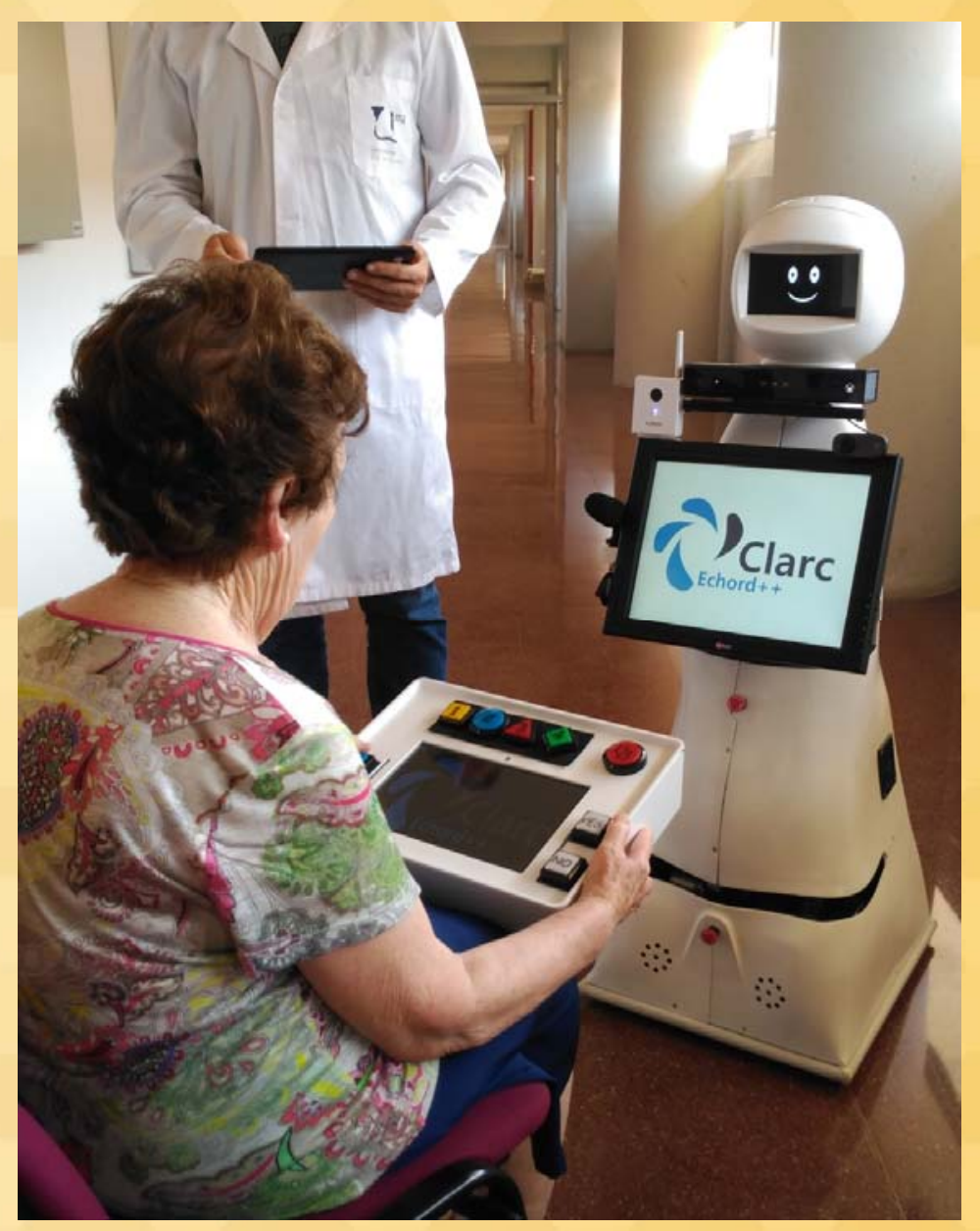




\section{The approach}

Active ageing

- requires continuous monitoring and assessment.

Comprehensive Geriatric Assessment (CGA)

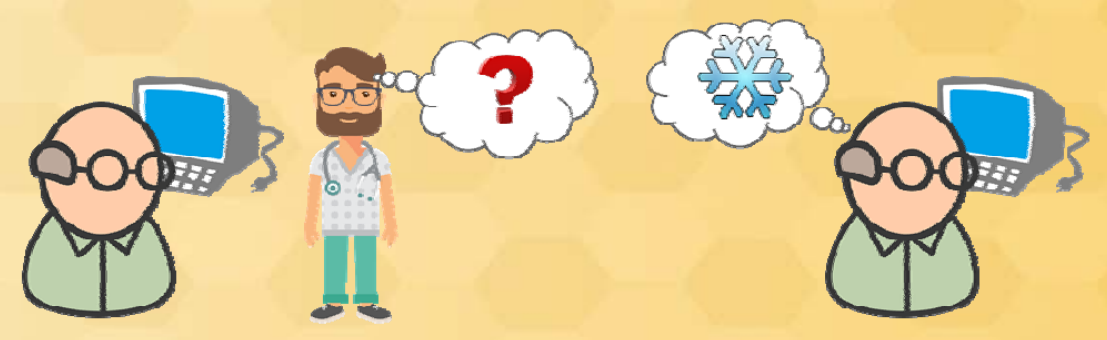

- Captures medical, psychosocial and functional capabilities and limitations.

- Includes different tests (e.g. Barthel, Minimental, Get Up \& Go)

- Some activities can be automated.

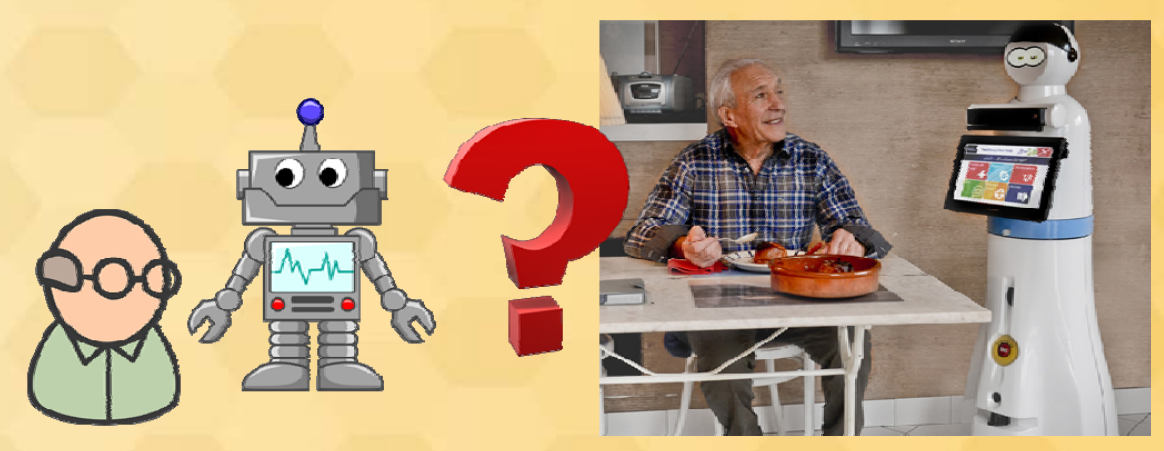

- ECHORD++ Project

- CLARC (FP7-ICT-601116).

- Assesstronic.

CLARC hypothesis: A social robot is the best tool to automate CGA procedures 


\section{The approach}

TecnologíaElectrónica

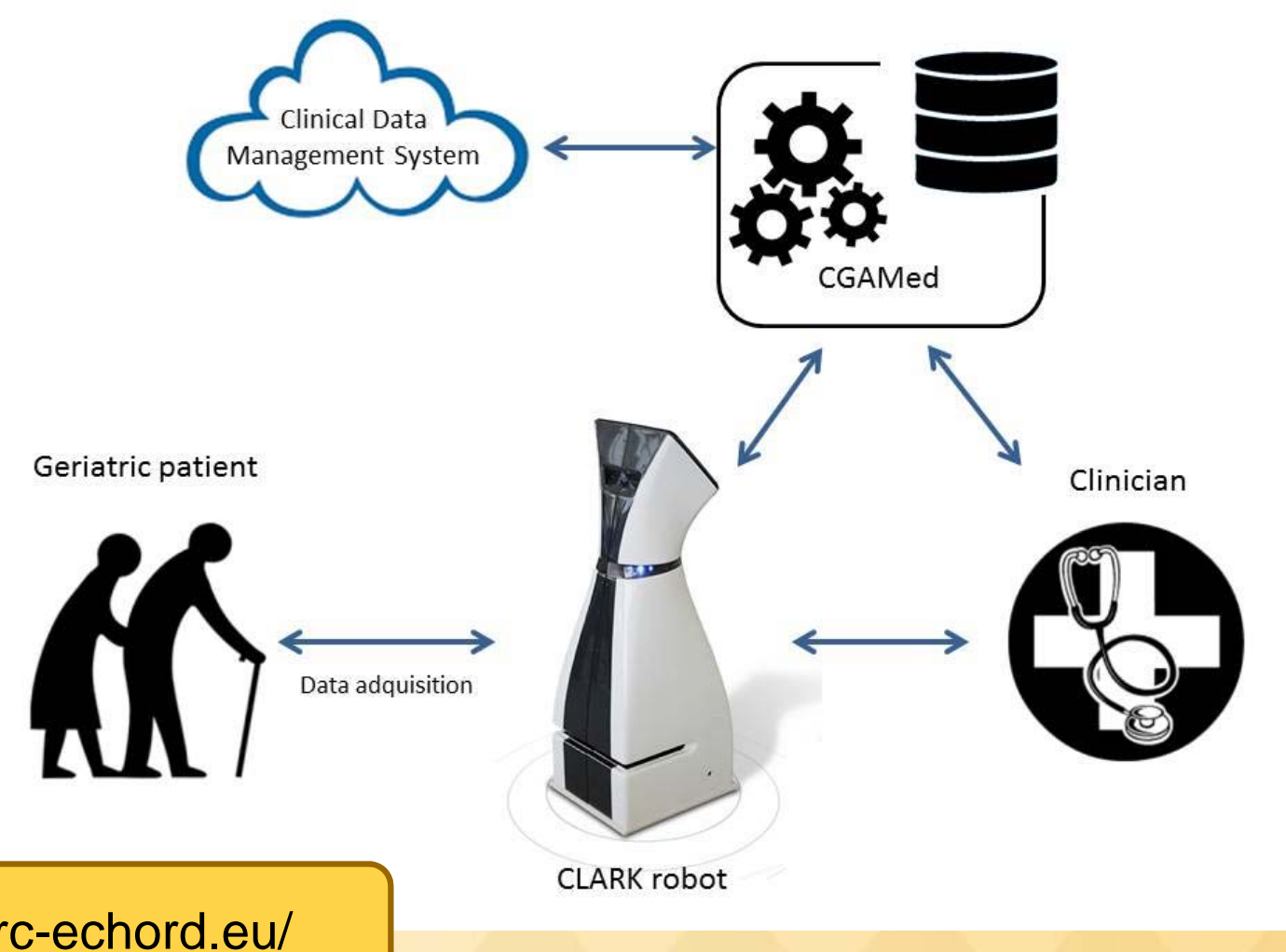

www.clarc-echord.eu/ 


\section{CLARC robot}

TecnologíaElectrónica
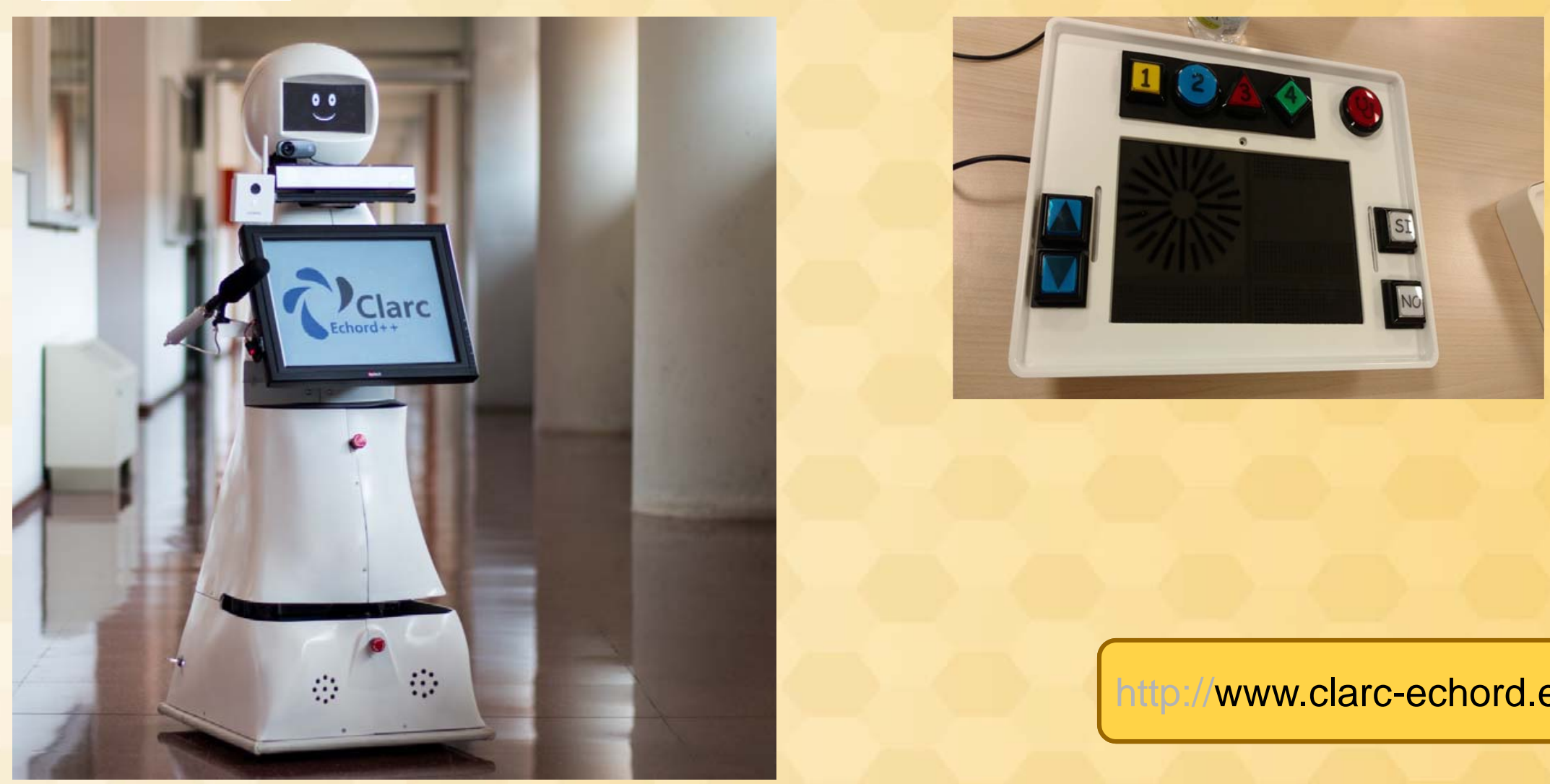

www.clarc-echord.eu/ 


\section{CLARC interfaces}

TecnologíaElectrónica

- For the person interacting with

- CGAMed for the clinician the robot
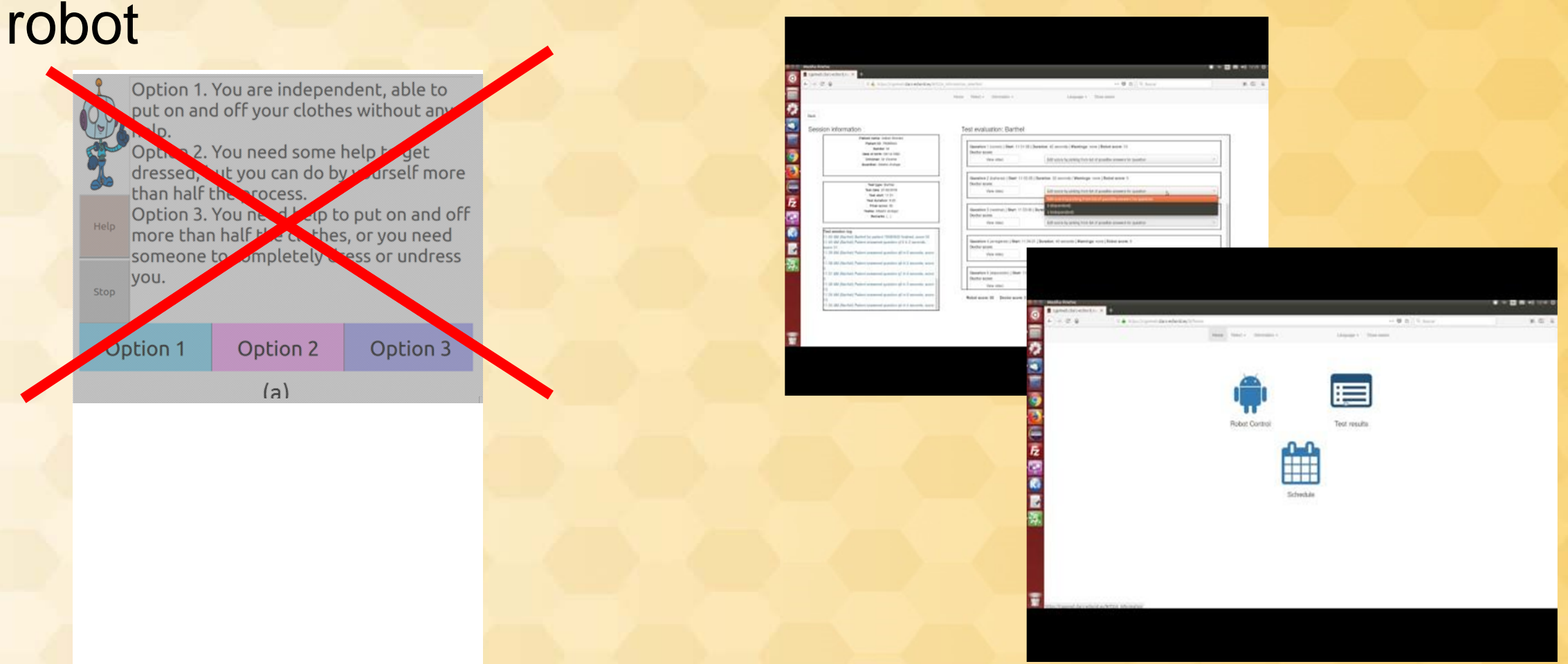


\section{Tests performed so far}

TecnologíaElectrónica

- Many technical and lab experiments

- Autonomous navigation

- Functional tests (Barthel): 13 users

- Cognitive tests (Minimental): 11 users

- Motor tests (Get Up \& Go): 15 users

- Autonomous tests

- Not completely autonomous use cases 


\section{CLARC: Phase III}

TecnologíaElectrónica

- 3 prototypes

- Retirement houses

- Hospital

- In France and Spain

- 1 robot for backup, update \& experimentation

- 3 months of testing

- Starting now! 


\section{Index}

TecnologíaElectrónica

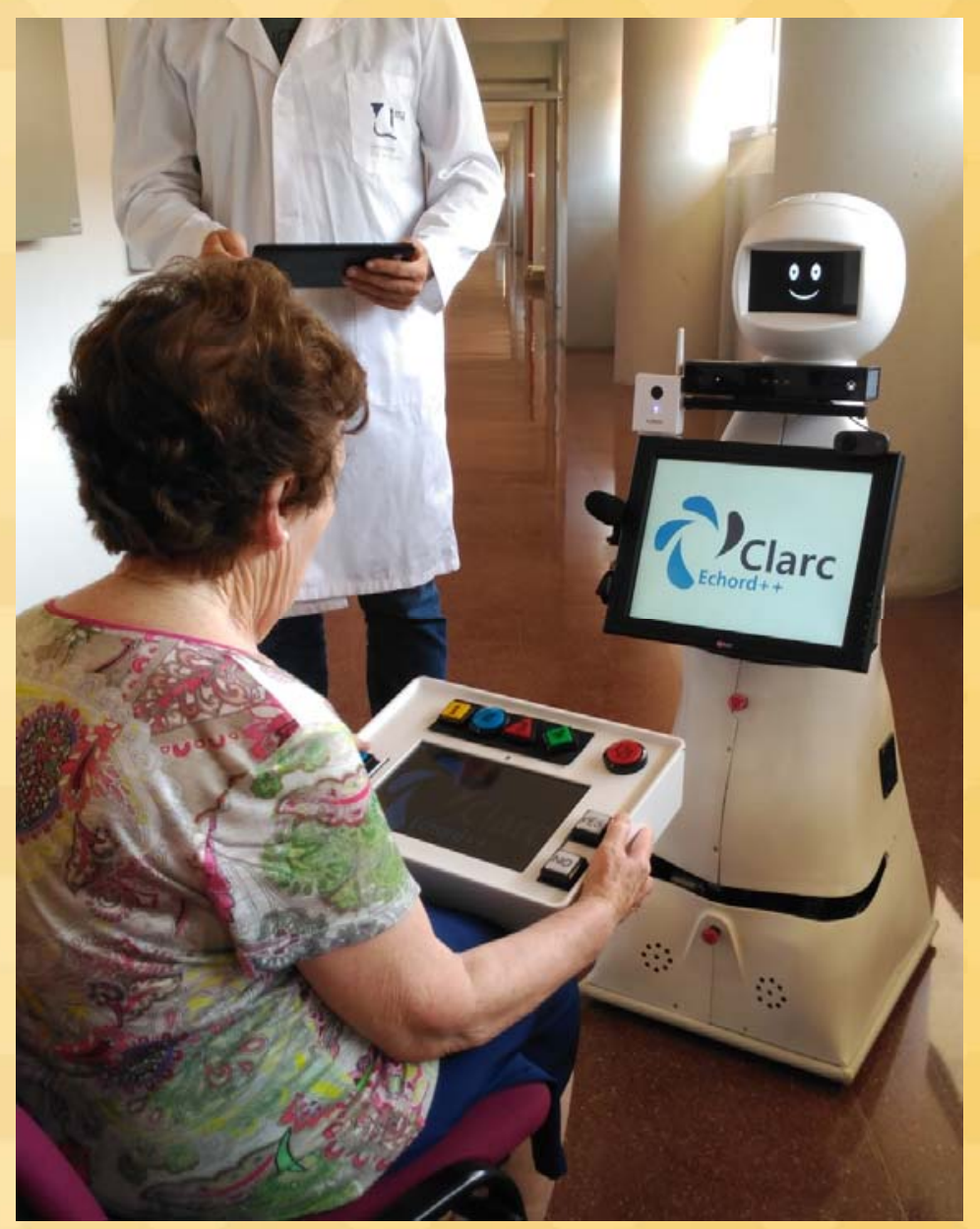




\section{Conclusion: What's a SAR good for?}

TecnologíaElectrónica

A SAR is a valuable tool:

- when interacting with groups of people

- when it saves time for the clinician

- when it can perform different tasks and adapt

- when it's accepted by the user

- Accesible, useful, trusted

- Functionality!

- when it's cheap enough
A SAR is not an option:

- in long-term one-to-one interactions (not right now)

- if it tries to replace human caregivers

- if it tries to be too emotive

- if it has to be too complex

- Safety

- Legal issues

- if it's too expensive 

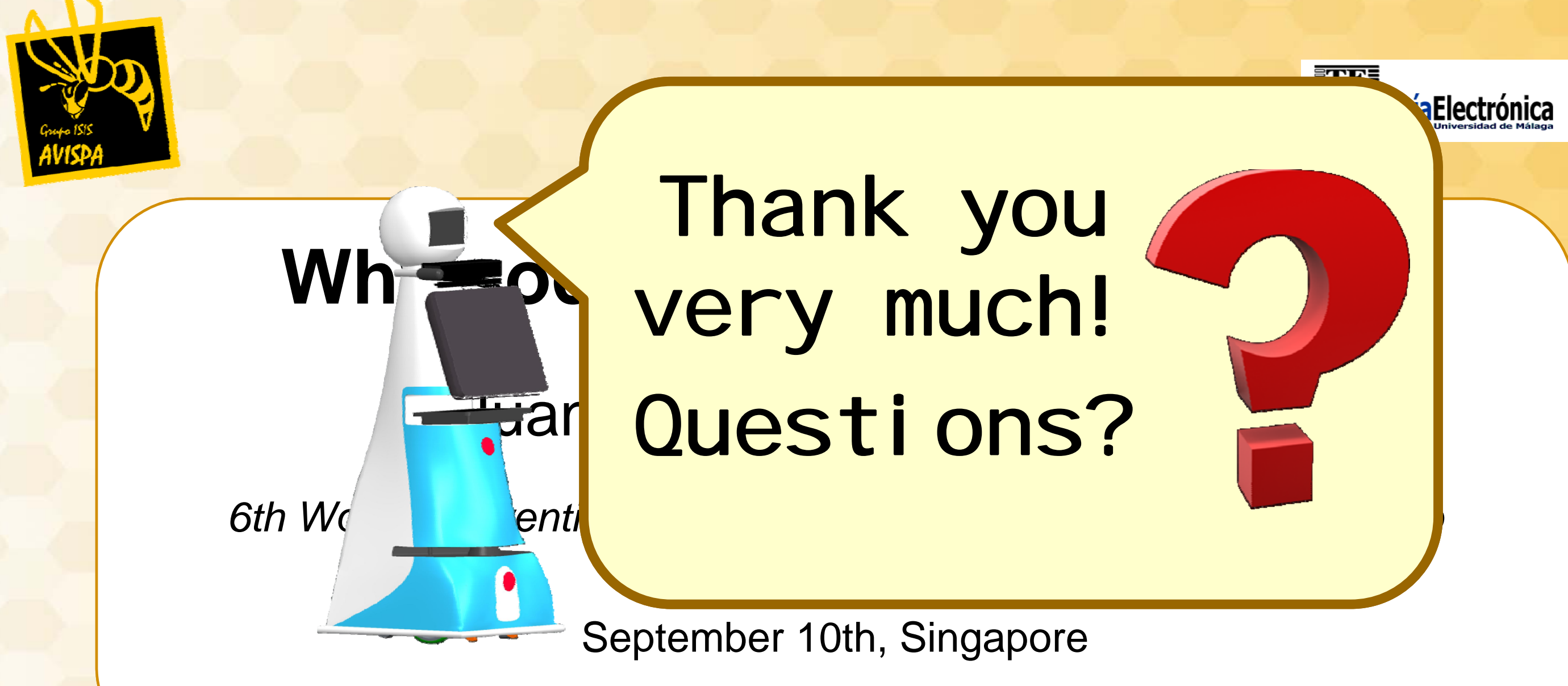